\title{
Priorización de las Dimensiones de Evaluación al Desempeño Docente por el Estudiante, en Tres Áreas del Conocimiento
}

\author{
Ernesto Cortés ${ }^{(1) \star}$, Margarita Campos ${ }^{(2)}$ y María P. Moreno ${ }^{(3)}$ \\ (1)Facultad de Cultura Física, Depto. de Posgrado, Benemérita Universidad Autónoma de Puebla, Ciudad \\ Universitaria s/n colonia Sn. Manuel, Puebla-México (e-mail: direcortes2001@yahoo.com.mx) \\ (2)Facultad de Medicina, Depto. de Quirúrgica, Benemérita Universidad Autónoma de Puebla, 13 sur 2709, \\ colonia Volcanes, Puebla-México (e-mail: armycampos@gmail.com) \\ (3)Facultad de Derecho y Ciencias Sociales, Benemérita Universidad Autónoma de Puebla, Ciudad \\ Universitaria s/n, colonia Sn. Manuel, Puebla-México, (e-mail: pmr1993@hotmail.com)
}

* Autor a quien debe ser dirigida la correspondencia

Recibido Oct. 3, 2013; Aceptado Dic.10, 2013; Versión final recibida Mar. 1, 2014

\section{Resumen}

Se presenta un estudio sobre la priorización de las dimensiones de evaluación al desempeño docente por el estudiante, en tres áreas del conocimiento. El estudio cubre el ciclo escolar 2009-2010, en el que se realiza la evaluación de la docencia por parte de los estudiantes de licenciatura de la Benemérita Universidad Autónoma de Puebla, en México. Esto se hace mediante un cuestionario formado por dimensiones que reflejan diversos aspectos y experiencias documentadas sobre la práctica docente que sustenta el Modelo Universitario Minerva. Miembros del Programa Institucional de Evaluación Académica toman al azar los resultados de las tres áreas del conocimiento, confrontándolos e identificando el orden de importancia que las dimensiones del cuestionario asumen durante ese periodo. La dimensión de Resultados del aprendizaje ocupa la primera posición. El estudiante aprecia y desea visualizar sus logros en el proceso enseñanza-aprendizaje y el docente que lo logra cubre la característica principal de la práctica docente exitosa, priorizada por los estudiantes.

\section{Prioritization of the Dimensions of Teacher Performance Evaluation by Students, in Three Areas of Knowledge}

\begin{abstract}
This paper presents a study on the prioritization of the dimensions of teacher performance evaluation by students, in three areas of knowledge. The study covers the period 2009-2010 in which the evaluation of teaching from the undergraduate student perspective was carried out at the Benemérita Universidad Autónoma de Puebla in Mexico. The evaluation was carried out by means of questionnaires which dimensions were based on the diverse aspects and documented experiences related to the practice of teaching that sustains the educational Minerva model of the university. Members of the Institutional Program for Academic Evaluation took the results from three areas of knowledge, chosen at random, and identified the order of importance taken by the dimensions of the questionnaire during this period. The dimension related to Results of Learning occupies the first place. The students value and want to visualize their achievements in the teaching-learning process and the teachers that achieve positive results fulfill the main characteristic of the successful practice of teaching prioritized by the students.
\end{abstract}

Keywords: teaching evaluation, evaluation by dimensions, educational model, successful teaching practice, results of learning 


\section{INTRODUCCIÓN}

En este artículo se presenta un análisis de los resultados de la evaluación que realizan los estudiantes hacia el desempeño docente mediante un instrumento basado en dimensiones en una universidad pública. En una primera intención se muestran los antecedentes y características del instrumento diseñado para un modelo educativo específico; en un segundo momento los criterios implementados para identificar las dimensiones que orientan la evaluación docente en siete Unidades Académicas (UA), organizadas en tres áreas del conocimiento del nivel superior de la BUAP. Asimismo, se describe la importancia que los estudiantes dan a cada dimensión, como una expresión particular de sus necesidades, finalmente, se enuncian algunas consideraciones especiales.

La evaluación de la docencia en la educación superior es necesaria como una respuesta a las exigencias de los estándares de calidad ante un mundo globalizado, competitivo y forzado a rendir cuentas en el gasto público (Van Vaugh, 1998). En España, la Agencia para la Calidad del Sistema Universitario de Catalunya (2001) menciona que la evaluación de la docencia se ha realizado por diversos procedimientos cómo: Autoevaluación del profesor; Evaluación por responsables académicos; Evaluación por los alumnos; Evaluación por colegas y Evaluación del aprendizaje de los estudiantes. Ante esta gran gama de posibilidades, Marsh concluye en una investigación de 30 años (1987-2007), que a nivel mundial se considera que, los estudiantes son fuente evaluadora confiable y válida de la eficacia del desempeño docente, por lo que la recomienda para los propósitos de mejorar la enseñanza, la toma de decisiones sobre el personal académico y salvaguardar el control de la calidad educativa.

Estos requerimientos y la confianza brindada al criterio de los estudiantes, han conducido a desarrollar instrumentos para la obtención de información sobre la calidad de la enseñanza, desde la evaluación tradicional basada en preguntas y respuestas, hasta la tendencia actual de la evaluación del desempeño docente basado en dimensiones, que implementa instrumentos o indicadores de las habilidades para: promover el aprendizaje de conocimientos por parte de los docentes, así como saber el grado de adquisición, toma de decisiones y mejora de los procesos de formación de los estudiantes (Rueda, 2009).

Ésta forma de evaluación, permite ubicar en la práctica docente aspectos como: la mediación y relación entre docentes y estudiantes y la obtención de información sobre toma de decisiones de tipo administrativo para la asignación de cursos, promociones y estímulos económicos (Luna y Torquemada, 2008)

En México la evaluación es fundamental, pues implica recursos y apoyos oficiales, por lo que se institucionalizó con el Programa para la Modernización Educativa 1989-1994, impulsando una mejora a la calidad de la educación superior a través de procesos de evaluación. Estas políticas educativas sometieron a las Instituciones de Educación Superior (IES) a procesos de rendición de cuentas ante organismos externos: Asociación Nacional de Universidades e Institutos de Educación Superior (ANUIES), entre otros, e internos: Vicerrectorías de Docencia, contraloría, Recursos humanos, etc.

La BUAP no quedó exenta de estos procesos e implementó un modelo educativo al que llamó Modelo Universitario Minerva (MUM), el cual es consecuencia de diferentes procesos de evaluación y revisión del actuar universitario como: “...el Proceso de Reforma Universitaria, Proyecto Fénix y el Proyecto Profesiones 2000, así como de la consulta realizada por el Honorable Consejo Universitario en 2004 (MUM, [DI]-2007-7). En estos procesos, resalta la evaluación de la docencia, sobre la cual la ANUIES, a la cual pertenece la BUAP, afirma: "la evaluación de la Docencia debe ser un objetivo prioritario en una Universidad,".

La BUAP, Desde el 2002, en concordancia con ANUIES, evalúa la docencia, y retoma entre las opciones consideradas por Catalunya y la experiencia de Marsh, la evaluación por los alumnos, lo que ha representado un ejercicio democrático, utilizando un instrumento de origen externo, para ésta evaluación se implementó el Programa Institucional de Evaluación Académica (PIEVA); siendo operado por docentes comisionados para ese efecto, de las distintas UA, los cuales fueron formados durante varios años en diversos aspectos (evaluación de la docencia, estadística, etc.), siendo coordinados por la Vicerrectoría de Docencia de la institución, sin embargo, al asumir el MUM fue necesario elaborar un instrumento del modelo asumido. Correspondió a una comisión del PIEVA la creación del instrumento PIEVA, siendo validado y piloteado en el año 2009, e implementado en el ciclo escolar 2010-2011, en el proceso de validación, participaron diversos investigadores internos y externos a la BUAP, así como el grupo PIEVA.

Previamente la Universidad de Baja California en México (2001), evaluó a los docentes por los alumnos, con un instrumento por dimensiones, sin embargo, condicionó el éxito a obtener cambios a posteriori en docentes evaluados deficientes, lo que no depende de la evaluación, sino de las acciones en consecuencia, además de que no priorizó las dimensiones. Los autores evalúan la docencia en siete Facultades organizadas en tres áreas del conocimiento, y a través del tratamiento estadístico a los resultados, priorizan 
las dimensiones, de la que es apreciada o más importante a la que es menos importante, bajo el precepto: si determinamos la dimensión más importante para los estudiantes del instrumento PIEVA, entonces, conoceremos la característica principal de la práctica docente exitosa priorizada por los estudiantes y lo que logra con los estudiantes de la BUAP, en las siete UA y en consecuencia de las tres áreas del conocimiento.

\section{MATERIALES Y MÉTODOS}

El instrumento PIEVA, permitió a la BUAP, realizar una evaluación interna de la docencia que tiene como eje central las actividades que el profesor desempeña en el salón de clases y su relación con los estudiantes, ésta es la que compete para esta investigación (Moreno, 1999). A través de ella se determinan y retroalimentan aspectos de la docencia requeridos por el MUM, el instrumento está organizado por siete dimensiones, con İtems de diversas opciones de respuesta, las que se designan: Relación, Mediación, Resultados del aprendizaje, Estrategias y Recursos, Planeación, Evaluación, y Ejes Transversales (éste último comprende: Formación Humana y Social, Desarrollo de habilidades del pensamiento complejo, Desarrollo de habilidades en el uso de Tecnología Información y Comunicación, Lengua extranjera y Desarrollo para emprendedores) se agregan algunos Ítems para la dimensión Generales Institucionales y una sección de comentarios abiertos. Estas dimensiones identificar y evalúan el desempeño docente y posteriormente, con base en los resultados, implementar acciones para: mejorar el aprendizaje, la planificación de la enseñanza, la organización de estrategias, el abordaje y aplicación de los conocimientos, los resultados y la evaluación asertiva de los procesos educativos (Alterio y Pérez, 2009). Ya Ellis menciona que la función docente es la ejecución de estas dimensiones para satisfacer necesidades del estudiante (1993 como se citó en Alterio y Pérez, 2009). El constructo se aprecia en la tabla 1.

\section{Tabla 1: Instrumento original de Evaluación Académica PIEVA-BUAP}

\begin{tabular}{|c|c|c|}
\hline Pregunta & Escala & Dimensión \\
\hline $\begin{array}{l}\text { Durante el desarrollo del presente curso, tu } \\
\text { profesor(a): Propicia un ambiente de confianza en el } \\
\text { aula }\end{array}$ & $\begin{array}{l}5 \text { Siempre } 4 \text { Casi siempre } 3 \text { A veces } 2 \\
\text { Ocasionalmente } 1 \text { Casi nunca }\end{array}$ & 1 Relación \\
\hline Favorece que ayudes a otros compañeros a aprender. & $\begin{array}{l}5 \text { Mucho, } 4 \text { Suficientemente, } 3 \text { Medianamente, } \\
2 \text { Insuficientemente, } 1 \text { Casi nunca }\end{array}$ & 1 Relación \\
\hline $\begin{array}{l}\text { Contribuye a incrementar tu compromiso con el } \\
\text { entorno (social, económico, ecológico) }\end{array}$ & $\begin{array}{l}5 \text { Mucho, } 4 \text { Suficientemente, } 3 \text { Medianamente, } \\
2 \text { Insuficientemente, } 1 \text { Casi nunca }\end{array}$ & 1 Relación \\
\hline Te motiva a aprender por ti mismo & $\begin{array}{l}5 \text { Mucho, } 4 \text { Suficientemente, } 3 \text { Medianamente, } \\
2 \text { Insuficientemente, } 1 \text { Casi nunca }\end{array}$ & 1 Relación \\
\hline Muestra disposición para resolver dudas. & $\begin{array}{l}5 \text { Siempre } 4 \text { Casi siempre } 3 \text { A veces } 2 \\
\text { Ocasionalmente } 1 \text { Casi nunca }\end{array}$ & 1 Relación \\
\hline $\begin{array}{l}\text { Tu maestro(a) te trata como persona, } \\
\text { independientemente del resultado académico }\end{array}$ & $\begin{array}{l}5 \text { Siempre } 4 \text { Casi siempre } 3 \text { A veces } 2 \\
\text { Ocasionalmente } 1 \text { Casi nunca }\end{array}$ & 1 Relación \\
\hline La claridad de las exposiciones en clase es: & $\begin{array}{l}5 \text { Muy buena } 4 \text { Buena, } 3 \text { regular, } 2 \text { Deficiente, } \\
1 \text { Muy deficiente }\end{array}$ & 2 Mediación \\
\hline $\begin{array}{l}\text { Las estrategias de enseñanza se adaptan a las } \\
\text { necesidades e intereses del grupo }\end{array}$ & $\begin{array}{l}5 \text { Siempre } 4 \text { Casi siempre } 3 \text { A veces } 2 \\
\text { Ocasionalmente } 1 \text { Casi nunca }\end{array}$ & 2 Mediación \\
\hline Al principio de un tema recupera lo que ya sabias & $\begin{array}{l}5 \text { Siempre } 4 \text { Casi siempre } 3 \text { A veces } 2 \\
\text { Ocasionalmente } 1 \text { Casi nunca }\end{array}$ & 2 Mediación \\
\hline $\begin{array}{l}\text { Plantea ideas diversas para generar en ti una postura } \\
\text { más activa. }\end{array}$ & $\begin{array}{l}5 \text { Totalmente de acuerdo, } 4 \text { De acuerdo, } 3 \mathrm{Ni} \\
\text { de acuerdo Ni en desacuerdo, } 2 \mathrm{En} \\
\text { desacuerdo, } 1 \text { Totalmente en desacuerdo }\end{array}$ & 2 Mediación \\
\hline Aprovecha los errores para promover el aprendizaje. & $\begin{array}{l}5 \text { Siempre } 4 \text { Casi siempre } 3 \mathrm{~A} \text { veces } 2 \\
\text { Ocasionalmente } 1 \text { Casi nunca }\end{array}$ & 2 Mediación \\
\hline $\begin{array}{l}\text { Durante este curso: } \\
\text { El trabajo en equipo coordinado por el(la) profesor(a) } \\
\text { involucra la participación de los integrantes }\end{array}$ & $\begin{array}{l}5 \text { Siempre } 4 \text { Casi siempre } 3 \text { A veces } 2 \\
\text { Ocasionalmente } 1 \text { Casi nunca }\end{array}$ & $\begin{array}{l}3 \text { Estrategias } \\
\text { y Recursos }\end{array}$ \\
\hline $\begin{array}{l}\text { Las estrategias utilizadas por el (la) profesor(a) facilitan } \\
\text { tu aprendizaje. }\end{array}$ & $\begin{array}{l}5 \text { Totalmente de acuerdo, } 4 \mathrm{De} \text { acuerdo, } 3 \mathrm{Ni} \\
\text { de acuerdo } \mathrm{Ni} \text { en desacuerdo, } 2 \mathrm{En} \\
\text { desacuerdo, } 1 \text { Totalmente en desacuerdo }\end{array}$ & $\begin{array}{l}3 \text { Estrategias } \\
\text { y Recursos }\end{array}$ \\
\hline $\begin{array}{l}\text { Las actividades planteadas utilizan plataformas web, } \\
\text { software básico o software específico. }\end{array}$ & $\begin{array}{l}5 \text { Siempre } 4 \text { Casi siempre } 3 \mathrm{~A} \text { veces } 2 \\
\text { Ocasionalmente } 1 \text { Casi nunca }\end{array}$ & $\begin{array}{l}3 \text { Estrategias } \\
\text { y Recursos }\end{array}$ \\
\hline La organización de los temas favorece mi aprendizaje. & $\begin{array}{l}5 \text { Mucho, } 4 \text { Suficientemente, } 3 \text { Medianamente, } \\
2 \text { Insuficientemente, } 1 \text { Casi nunca }\end{array}$ & 4 Planeación \\
\hline $\begin{array}{l}\text { El tiempo asignado a cada tema permite el logro de } \\
\text { sus objetivos de aprendizaje }\end{array}$ & $\begin{array}{l}5 \text { Totalmente de acuerdo, } 4 \text { De acuerdo, } 3 \mathrm{Ni} \\
\text { de acuerdo Ni en desacuerdo, } 2 \text { En } \\
\text { desacuerdo, } 1 \text { Totalmente en Desacuerdo } \\
\text { (TD) }\end{array}$ & 4 Planeación \\
\hline La evaluación corresponde a lo que se enseña. & $\begin{array}{l}5 \text { Totalmente de acuerdo, } 4 \text { De acuerdo, } 3 \mathrm{Ni} \\
\text { de acuerdo Ni en desac., } 2 \text { En desac., } 1 \text { TD }\end{array}$ & 5 Evaluación \\
\hline
\end{tabular}




\section{Tabla 1: Continuación}

Los criterios para evaluar se aplican de igual forma para todos

Las actividades propuestas en esta materia favorecen la búsqueda y selección de información como una actividad de investigación

Las actividades y tareas propuestas favorecen el empleo de una segunda lengua

En qué medida el profesor te ayuda a:

A) Reflexionar sobre los temas abordados determinando lo que sabes y hasta dónde llegan tus conocimientos

B) Defender tus conocimientos en relación a otras posturas; argumentar sobre tus puntos de vista y los de otras personas

C) Plantearte nuevas teorías

El trabajo realizado por tu profesor(a) facilita que: Relaciones los contenidos del curso con otras materias Identifiques la forma en que aprendes mejor

Que mejores tu desempeño al plantearte diversos retos intelectuales.

Tu expresión oral y escrita mejoren

Las actividades del curso te han permitido practicar lo aprendido en clase

Durante este curso:

¿A qué porcentaje de sesiones programadas ha asistido tu profesor(a)?

Del total de sesiones programadas, ¿En qué porcentaje ha cumplido tu profesor(a) con el horario establecido?

El dominio de los contenidos de la materia que el profesor(a) imparte es:

La cobertura del profesor(a) de los temas

contemplados en el programa es de un:

Con lo abordado hasta hoy ¿Consideras que se han

logrado los objetivos de aprendizaje del curso?
5 Totalmente de acuerdo, $4 \mathrm{De}$ acuerdo, $3 \mathrm{Ni}$ de acuerdo $\mathrm{Ni}$ en desacuerdo, $2 \mathrm{En}$ desacuerdo, 1 Totalmente en Desacuerdo 5 Totalmente de acuerdo, 4 De acuerdo, $3 \mathrm{Ni}$ de acuerdo $\mathrm{Ni}$ en desacuerdo, $2 \mathrm{En}$ desacuerdo, 1 Totalmente en Desacuerdo 5 mucho, 4 suficientemente, 3 medianamente, 2 insuficientemente, 1 Casi Nada (CN)

5 Mucho, 4 Suficientemente, 3

Medianamente, 2 Insuficientemente, $1 \mathrm{CN}$

5 mucho, 4 suficientemente, 3

medianamente, 2 insuficientemente, $1 \mathrm{CN}$

5 mucho, 4 suficientemente, 3 medianamente, 2 insuficientemente, $1 \mathrm{CN}$

5 Mucho, 4 Suficientemente, 3

Medianamente, 2 Insuficientemente, $1 \mathrm{CN}$

5 Totalmente de acuerdo, 4 De acuerdo, $3 \mathrm{Ni}$ de acuerdo $\mathrm{Ni}$ en desacuerdo, $2 \mathrm{En}$ desacuerdo, 1 Totalmente en Desacuerdo

5 mucho, 4 suficientemente, 3

medianamente, 2 insuficientemente, $1 \mathrm{CN}$

5 mucho, 4 suficientemente, 3

medianamente, 2 insuficientemente, $1 \mathrm{CN}$

5 mucho, 4 suficientemente, 3

medianamente, 2 insuficientemente, $1 \mathrm{CN}$

$100 \%, 90 \%, 80 \%, 70 \%, 60 \%$ o menos

$100 \%, 90 \%, 80 \%, 70 \%, 60 \%$ o menos

5 Muy bueno 4 bueno, 3 regular, 2 deficiente, 1 Muy deficiente

$100 \%, 90 \%, 80 \%, 70 \%, 60 \%$ o menos

5 Mucho, 4 Suficientemente, 3

Medianamente, 2 Insuficientemente, $1 \mathrm{CN}$
5 Evaluación

6 Ejes

Transversales

6 Ejes

Transversales

6 Ejes

Transversales

6 Ejes

Transversales

6 Ejes

Transversales

7 Resultados

del Aprendizaje

7 Resultados

del Aprendizaje

7Resultados

del Aprendizaje

7 Resultados del Aprendizaje

7 Resultados del Aprendizaje

Generales

Institucionales

Generales

Institucionales

Generales Institucionales

Generales Institucionales Generales Institucionales

El proceso evaluativo, se realizó en 2010 y 2011, en los periodos primavera, verano y otoño, dos meses después de iniciado el curso escolar, cuando los estudiantes tienen conocimiento de las actitudes y aptitudes de los docentes en asignatura y sección impartida. La difusión se realizó mediante la página BUAP y por carteles en cada UA, garantizando que los estudiantes conocieran las fechas de evaluación.

Tabla 2: Número de evaluaciones, asignaciones, porcentaje de cobertura y Alfa de Cronbach.

\begin{tabular}{|l|c|c|c|c|c|c|c|}
\hline & \multicolumn{2}{|c|}{$\begin{array}{c}\text { Número de } \\
\text { Evaluaciones }\end{array}$} & \multicolumn{2}{c|}{$\begin{array}{c}\text { Evaluaciones } \\
\text { Asignadas }\end{array}$} & \multicolumn{2}{c|}{$\%$ de cobertura } & \multirow{2}{*}{$\begin{array}{c}\text { Alfa de } \\
\text { Cronbach }\end{array}$} \\
\hline Unidad Académica & 2011 & 2010 & 2011 & 2010 & 2011 & 2010 & 0.981 \\
\hline Comunicación & 5394 & 5142 & 10360 & 9570 & $52.07 \%$ & $53.73 \%$ & 0.982 \\
\hline Contaduría pública & 30296 & 31856 & 42098 & 40384 & $71.97 \%$ & $78.88 \%$ & 0.978 \\
\hline Cultura física & 7066 & 6251 & 11402 & 10639 & $61.97 \%$ & $58.76 \%$ & 0.978 \\
\hline Enfermería & 4592 & 5529 & 6338 & 7125 & $72.45 \%$ & $77.60 \%$ & 0.985 \\
\hline Filosofía y letras & 7855 & 9525 & 14157 & 12747 & $55.48 \%$ & $74.72 \%$ & 0.984 \\
\hline Medicina & 14982 & 18270 & 42095 & 39806 & $35.59 \%$ & $45.90 \%$ & 0.982 \\
\hline Psicología & 3462 & 2399 & 16454 & 11934 & $21.04 \%$ & $20.10 \%$ & 0.970 \\
\hline Total & 75658 & 80982 & 144915 & 134215 & $52.21 \%$ & $60.34 \%$ & 0.982 \\
\hline
\end{tabular}


Contestando voluntariamente, en línea (como una forma de respeto al medio ambiente), en la sección autoservicios de la página BUAP; cada estudiante ingresó con su número de matrícula y una contraseña. EI proceso duró dos semanas y fue confidencial, la participación se consideró adecuada de acuerdo al medio en que se desarrolló, siendo el margen de error del $0.22 \%$, la información se presenta en la tabla 2.

Para conocer la medida de la adecuación muestral, se aplica el coeficiente de Kaiser-Meyer-Olkin (Coeficiente KMO), resultando: Excelente adecuación muestral $(0,9<\mathrm{KMO} 1,0)$, así como una Prueba de esfericidad de Bartlett, para conocer si la matriz de correlaciones es una matriz identidad, lo cual indicaría que el modelo factorial es inadecuado. El estadístico de Bartlett se obtiene a partir de una transformación $\mathrm{X}^{2}$ del determinante de la matriz de correlaciones y cuanto mayor sea, y por tanto menor el nivel de significación, más improbable es que la matriz sea una matriz identidad y más adecuado resulta el análisis factorial, los resultados se presentan en la tabla 3.

Tabla 3: KMO y prueba de Bartlett

\begin{tabular}{|c|c|c|c|c|c|c|c|c|}
\hline & \multicolumn{7}{|c|}{ Unidad académica } \\
\hline & & $\begin{array}{l}\text { Comuni- } \\
\text { cación }\end{array}$ & $\begin{array}{l}\text { Contaduría } \\
\text { Pública }\end{array}$ & $\begin{array}{l}\text { Cultura } \\
\text { física }\end{array}$ & Enfermería & $\begin{array}{c}\text { Filosofía y } \\
\text { Letras }\end{array}$ & Medicina & Psicología \\
\hline \multicolumn{2}{|c|}{$\begin{array}{l}\text { Medida de adecuación } \\
\text { muestral de Kaiser- } \\
\text { Meyer-Olkin .990 }\end{array}$} & 0.987 & 0.989 & 0.987 & 0.988 & 0.989 & 0.989 & 0.962 \\
\hline \multirow{3}{*}{$\begin{array}{l}\text { Prueba de } \\
\text { esfericidad } \\
\text { de Bartlett }\end{array}$} & \begin{tabular}{|l|}
$\begin{array}{l}\text { Chi- } \\
\text { cuadrado } \\
\text { aproximado } \\
1337143.861\end{array}$ \\
\end{tabular} & 84752.9 & 594699.0 & 78152.6 & 117479.6 & 161576.3 & 298274.6 & 5546.2 \\
\hline & Gl 406 & 406 & 406 & 406 & 406 & 406 & 406 & 406 \\
\hline & \begin{tabular}{|l|l} 
Sig. .000 \\
\end{tabular} & 0 & 0 & 0 & 0 & 0 & 0 & 0 \\
\hline
\end{tabular}

Con los resultados se construyó una base de datos, aplicando tratamiento estadístico mediante la paquetería Statistical Package for the Social Sciencs (SPSS V17), con análisis de componentes principales (ACP), el cual permite la aglomeración de ítems alrededor de otros con mayor significancia, conformando nuevos grupos, permitiendo cuando se consideró pertinente, reasignar el nombre por dimensión siendo: Resultados, Relación, Mediación, Estrategias de Enseñanza, Planeación, Evaluación de Aprendizajes y Ejes Transversales. También se identifica el orden de importancia que los estudiantes dan a las dimensiones, ordenando de lo que consideran más relevante a lo menos relevante, permitiendo que el orden de importancia fuera diferente para cada UA y área del conocimiento, como se observa en la Tabla 4.

Tabla 4: Los asteriscos señalan la agrupación de ítems mediante análisis de componentes principales en la Facultad de Medicina BUAP.

\begin{tabular}{|c|c|c|}
\hline Componente & Rotados con sus Variables & Dimensión Original \\
\hline \multirow{9}{*}{1} & C) Plantearte nuevas teorías & Eje Transversal \\
\hline & Que mejores tu desempeño al plantearte diversos retos intelectuales. ${ }^{*}$ & Resultados \\
\hline & Relaciones los contenidos del curso con otras materias & Resultados \\
\hline & $\begin{array}{l}\text { B) Defender tus conocimientos en relación a otras posturas; argumentar sobre } \\
\text { tus puntos de vista y los de otras personas }\end{array}$ & Eje Transversal \\
\hline & Identifiques la forma en que aprendes mejor & Resultados \\
\hline & Tu expresión oral y escrita mejoren & Resultados \\
\hline & $\begin{array}{l}\text { A) Reflexionar sobre los temas abordados determinando lo que sabes y hasta } \\
\text { dónde llegan tus conocimientos }\end{array}$ & Eje Transversal \\
\hline & Las actividades del curso te han permitido practicar lo aprendido en clase & Resultados \\
\hline & $\begin{array}{l}\text { Las actividades propuestas en esta materia favorecen la búsqueda y selección } \\
\text { de información como una actividad de investigación }\end{array}$ & Eje Transversal \\
\hline \multirow{8}{*}{2} & $\begin{array}{l}\text { Las estrategias de enseñanza se adaptan a las necesidades e intereses del } \\
\text { grupo }\end{array}$ & Mediación \\
\hline & Al principio de un tema recupera lo que ya sabias & Mediación \\
\hline & La claridad de las exposiciones en clase es: & Mediación \\
\hline & Las estrategias utilizadas por el (la) profesor(a) facilitan tu aprendizaje. & Estrategias y Recursos \\
\hline & Plantea ideas diversas para generar en ti una postura más activa. ${ }^{*}$ & Mediación \\
\hline & La organización de los temas favorece mi aprendizaje. & Planeación \\
\hline & Aprovecha los errores para promover el aprendizaje. & Mediación \\
\hline & Te motiva a aprender por ti mismo & Relación \\
\hline
\end{tabular}


Tabla 4: Continuación

\begin{tabular}{|c|c|c|}
\hline \multirow{6}{*}{3} & $\begin{array}{l}\text { Tu maestro(a) te trata como persona, independientemente del resultado } \\
\text { académico }\end{array}$ & Relación \\
\hline & Propicia un ambiente de confianza en el aula & Relación \\
\hline & Muestra disposición para resolver dudas. & Relación \\
\hline & Favorece que ayudes a otros compañeros a aprender.* & Relación \\
\hline & $\begin{array}{l}\text { Contribuye a incrementar tu compromiso con el entorno (social , económico, } \\
\text { ecológico) }\end{array}$ & Relación \\
\hline & Los criterios para evaluar se aplican de igual forma para todos & Evaluación \\
\hline \multirow{3}{*}{4} & La evaluación corresponde a lo que se enseña. & Evaluación \\
\hline & Las evaluaciones constantes me orientan sobre el avance logrado en la materia* & Evaluación \\
\hline & El tiempo asignado a cada tema permite el logro de sus objetivos de aprendizaje & Planeación \\
\hline 5 & $\begin{array}{l}\text { Las actividades planteadas utilizan plataformas web, software básico o software } \\
\text { específico.* }\end{array}$ & Estrategias y Recursos \\
\hline 6 & $\begin{array}{l}\text { Las actividades y tareas propuestas favorecen el empleo de una segunda } \\
\text { lengua.* }\end{array}$ & Eje Transversal \\
\hline 7 & $\begin{array}{l}\text { El trabajo en equipo coordinado por el (la) profesor(a) involucra la participación } \\
\text { de los integrantes. }\end{array}$ & Estrategias y Recursos \\
\hline
\end{tabular}

En lo particular, cada cuatrimestre, los docentes recibieron el resultado de sus evaluaciones, ingresando a la página BUAP, autoservicios, conociendo aciertos y debilidades de su cátedra frente a los estudiantes.

En lo general, los autores confrontaron la información obtenida, identificando las dimensiones que coinciden o difieren y su orden en los resultados, así como su organización en forma global de siete UA, organizadas en tres áreas del conocimiento. Las seleccionadas son: Área de la salud (Facultad de Medicina FM, Facultad de Enfermería FE, Facultad de Cultura Física FCF), Económico Administrativas (Facultad de Contaduría FCON, Facultad de Ciencias de la Comunicación FCOM), Área de Educación y Humanidades (Facultad de Psicología FP, Facultad de Filosofía y Letras FFL), estas fueron escogidas al azahar.

\section{RESULTADOS Y DISCUSIÓN}

Los resultados por UA, se presentan en la tabla 5 y se analizan bajo dos formas: Análisis por programas educativos organizados por área del conocimiento Análisis por resultados globales y significado de dimensiones

Tabla 5: Concentrado de Dimensiones del Análisis de Componentes Principales, resultados agrupados por área del conocimiento y análisis global

\begin{tabular}{|c|c|c|c|c|c|c|c|}
\hline \multirow{2}{*}{$\begin{array}{l}\text { Área del } \\
\text { conocimiento } \\
\text { Unidad } \\
\text { Académica } \rightarrow \\
\text { Dimensiones por } \\
\text { orden de } \\
\text { importancia } \downarrow\end{array}$} & \multicolumn{2}{|c|}{$\begin{array}{l}\text { Área Económico } \\
\text { Administrativas }\end{array}$} & \multicolumn{3}{|c|}{ Área de la Salud } & \multicolumn{2}{|c|}{$\begin{array}{l}\text { Área de Educación y } \\
\text { Humanidades }\end{array}$} \\
\hline & $\begin{array}{l}\text { Comunicación } \\
\text { FCOM }\end{array}$ & $\begin{array}{l}\text { Contaduría } \\
\text { FCON }\end{array}$ & $\begin{array}{l}\text { Cultura } \\
\text { Física } \\
\text { FCF }\end{array}$ & $\begin{array}{l}\text { Enfermería } \\
\text { FE }\end{array}$ & $\begin{array}{l}\text { Medicina } \\
\text { FM }\end{array}$ & $\begin{array}{l}\text { Filosofía y } \\
\text { Letras. } \\
\text { FFL }\end{array}$ & $\begin{array}{l}\text { Psicología } \\
\text { FP }\end{array}$ \\
\hline 1 & Resultados & Resultados & Resultados & Resultados & Resultados & Resultados & Resultados \\
\hline 2. & Relación & Mediación & $\begin{array}{l}\text { Escenario } \\
\text { de } \\
\text { Aprendizaje }\end{array}$ & Relación & \begin{tabular}{|l|} 
Escenario \\
de \\
Aprendizaje
\end{tabular} & \begin{tabular}{|l|} 
Escenario \\
de \\
Aprendizaje
\end{tabular} & $\begin{array}{l}\text { Escenario } \\
\text { de } \\
\text { Aprendizaje }\end{array}$ \\
\hline 3 & Mediación & Evaluación & Evaluación & $\begin{array}{l}\text { Escenario } \\
\text { de } \\
\text { Aprendizaje }\end{array}$ & Relación & Relación & Relación \\
\hline 4 & Planeación & Relación & Actitud & Evaluación & Evaluación & Evaluación & Evaluación \\
\hline 5 & Evaluación & Actitud & Relación & Uso de TIC & Uso de TIC & Uso de TIC & Planeación \\
\hline 6 & $\begin{array}{l}\text { Segunda } \\
\text { Lengua }\end{array}$ & $\begin{array}{l}\text { Segunda } \\
\text { Lengua }\end{array}$ & $\begin{array}{l}\text { Eje } \\
\text { Transversal }\end{array}$ & $\begin{array}{l}\text { Segunda } \\
\text { Lengua }\end{array}$ & $\begin{array}{l}\text { Segunda } \\
\text { Lengua }\end{array}$ & Planeación & Mediación \\
\hline 7 & Uso de TIC & $\begin{array}{l}\text { Uso de } \\
\text { TIC }\end{array}$ & Clima & & Planeación & \begin{tabular}{|l|}
$\begin{array}{l}\text { Segunda } \\
\text { Lengua }\end{array}$ \\
\end{tabular} & $\begin{array}{l}\text { Segunda } \\
\text { Lengua }\end{array}$ \\
\hline
\end{tabular}

Análisis por programas educativos organizados por área del conocimiento. Destaca en primer lugar la dimensión Resultados del aprendizaje, como la más relevante y por lo tanto la más valorada, tanto en las siete UA, como en las tres áreas del conocimiento, indicando que los estudiantes, consideran que es la dimensión a priorizar por un docente para considerarlo exitoso. El área del conocimiento más consistente es 
la de Educación y Humanidades, coincidiendo en orden de mayor a menor importancia cuatro dimensiones: Resultados, Escenario de aprendizaje, Relación, Evaluación y a partir de ese momento se observan divergencias. La siguiente área con mayor consistencia es la de Salud, coincidiendo en dos UA (FCF y FM), las dimensiones de Resultados y escenarios de aprendizaje, iniciando a partir de ese momento diferencias. En el área Económico Administrativas, desde el segundo se observan diferencias: Relación y Mediación.

Análisis por resultados globales y significado de dimensiones: La agrupación de ítems 1 en todas las UA fue: Resultados del aprendizaje. Representa la característica principal para identificar a un académico sobresaliente, es el que: logra aprendizaje desde que inicia el ciclo escolar hasta que lo concluye, siendo esto, resultado del desempeño del académico a través del desarrollo de actividades sustantivas y del seguimiento que éste da a las mismas.

El segundo lugar en importancia presenta diferencias. Las FCF, FM, FFL y FP ubican aquí a los Escenarios de Aprendizaje, lo que representa que el académico genera motivación por medio de: un entorno que no es más que el sitio en donde se va a desarrollar el aprendizaje de conocimientos, un ambiente activo y un clima favorable mediado por estrategias. (Duarte 2003) En contraste, la FCOM y FE consideran a la dimensión de Relación docente estudiante, por: el rol docente que desempeña el académico (colaborador, tutor, guía, organizador, facilitador con humanismo) y un rol del estudiante que se asigna para desarrollar todas las habilidades y competencias para el trabajo. La FCON coloca en esta segunda agrupación a la Mediación considerada por Ahumada (2005) como "el Comportamiento diferente, preocupación y ayuda que cada docente emplea para la adquisición de saberes y su aplicación" es decir la capacidad del docente de adaptar el conocimiento y la forma en que será proporcionado de acuerdo a las características del grupo.

En la agrupación de los ítems 3, las FM, FP y FFL identifican a la dimensión de Relación, en cambio las FCF y FCON ubican a la dimensión de Evaluación, seguramente debido a que: los estudiantes tienen necesidad de saber cómo avanzan académicamente y si van aprobar la materia. En el caso de la FE ubica a la dimensión de Escenario de Aprendizaje ya que destaca la alta correlación entre los conocimientos que adquieren y la práctica profesional realizada por los estudiantes en el lugar de trabajo.

La agrupación de ítems 4 para las FE, FM, FFL y FP es la dimensión de Evaluación. En el área EconómicoAdministrativa, se presentan diferencias ya que la FCON tiene la dimensión de Relación. En la FCOM es la dimensión de Planeación la que: denota la importancia que tiene para los estudiantes la preparación de las actividades que el académico lleve a cabo en el aula. Finalmente en la FCF, la cuarta dimensión es la Actitud docente que: identifica la capacidad del docente para transmitir sus conocimiento sin importar la condición tanto intelectual o social del estudiante.

En las agrupaciones 5, 6 y 7 de los ítems restantes, se observó un comportamiento semejante entre las dimensiones, que osciló mayoritariamente entre el manejo de las TIC's, una Segunda Lengua, y Ejes Transversales, producto de la redimensión que permite el tratamiento estadístico del instrumento, aparece el Clima laboral, entendiéndose por este: la importancia que otorgan los estudiantes a las buenas relaciones que esperan entre estudiantes y académicos, pero enriquecida por las relaciones con el resto del personal (administrativos, trabajadores no académicos, etc.), la Actitud también se manifiesta en Área Económico Administrativas, concretamente en la FCON. En la FE, el análisis estadístico solo reportó seis dimensiones, de acuerdo a la variabilidad y comportamiento de sus datos, por lo que no se especifica una séptima dimensión. Al obtener los resultados, se observa que el conjunto de variables que arroja el ACP queda integrado de diferente forma al de Análisis Factorial Exploratorio (AFE), ya que el método de extracción o factorización es distinto y la elección del método adecuado dependerá del enfoque o estudio que se quiera realizar, el cual permite el ajuste de las saturaciones (nivel de pertenencia a cada componente).

\section{CONCLUSIONES}

1.- Se corroboró la factibilidad de aplicación del constructo a partir de la participación de los estudiantes, así como su tratamiento estadístico y consecuente redimensión de las prioridades del desempeño docente por área del conocimiento y programa educativo, detectando que existen semejanzas y diferencias en el orden de importancia de las dimensiones que conforman el instrumento en las tres áreas del conocimiento y de las siete UA.

2.- De acuerdo a la opinión de los estudiantes, la dimensión que priorizan o consideran la más importante es la de: Resultados del aprendizaje, por lo que es la característica principal para identificar a un académico sobresaliente, dando cumplimiento al primer propósito de la investigación. 
3.- A partir de las variables coincidentes, las características que prevalecen y orientan el desempeño docente exitoso, en orden de importancia son: Resultados, Escenarios de Aprendizaje, Relación y Evaluación. Las dimensiones quinta, sexta y séptima, gravitan entre el manejo de TIC, una segunda lengua, así como de los ejes transversales, planeación y mediación. Producto del tratamiento estadístico y redimensión de los resultados, se incorpora Actitud y el Clima Institucional. Con esto se da cumplimiento al propósito de priorizar el orden de las dimensiones en las tres áreas del conocimiento y en forma general, apreciándose que las diferencias seguramente están en relación a la formación en las diversas profesiones, tanto positivas como negativas. El principal aporte es que la investigación revela que al estudiante principalmente le interesa lo que logra el docente con ellos, y secundariamente otros aspectos, lo que resulta novedoso, pues la mayoría de las evaluaciones, se centran en lo que hace el docente, no en sus logros con el estudiante, con esta conclusión, se da por concluida esta investigación.

\section{REFERENCIAS}

Ahumada, A. P., "Hacia una evaluación auténtica del aprendizaje", 22. México: Paidós, Ecuador, (2005).

Alterio, A. GH y Pérez, L. HL. "Evaluación de la función docente según el desempeño de los profesores y la opinión estudiantil”. Revista electrónica de Educación Médica Superior. ISSN: 0864-2141 (en línea) 23(3) http://scielo.sld.cu/scielo.php?script=sci_arttext\&pid=S0864-21412009000300001, (2009).

ANUIES "Programa Nacional de Superación del Personal Académico", Revista Confluencia. No. Extraordinario, año 2, (1994).

BUAP Vicerrectoría de Docencia "Principales rasgos de la evaluación académica desde el enfoque del constructivismo en el Programa Institucional de Evaluación al Académico, documento inédito. Puebla, México. (2008).

BUAP Vicerrectoría de Docencia "Fundamentos Modelo Universitario Minerva", documento inédito. Puebla, México. (2007)

Luna, S. E. y Rueda, B. M., "Participación de académicos y estudiantes en la evaluación de la docencia". Revista Perfiles Educativos ISSN: 0185-2698 (en línea) XXIII (93) [Fecha de consulta: 7 de noviembre de 2013] Disponible en:<http://redalyc.org/articulo.oa?id=13209302> pp. 7-27, Instituto de Investigaciones sobre la Universidad y la Educación México, (2001).

Luna, E. y Torquemada, A., "Los cuestionarios de evaluación de la docencia por los alumnos: balance y perspectivas de su agenda". Revista Electrónica de Investigación Educativa Especial. Número especial ISSN: 1607-4041 (en línea) http://redie.uabc.mx/NumEsp1/contenido-lunatorquemada.html, (2008).

Marsh, H. W., Students' evaluations of university teaching: A multidimensional perspective. In R. P. Perry y J C. Smart (Ed.), The scholarship of teaching and learning in higher education: An evidence-based perspective (en línea) (pp.319-384). New York: Springer. http://www.education.ox.ac.uk/research/self/research/studentevaluations-of-teaching-effectiveness/, (2007).

Messina, G., "Investigación en o investigación acerca de la formación docente: un estado del arte en los noventa" Revista Iberoamericana de Educación. No. (19), Enero - Abril (1999).

Rueda, B. M., La evaluación del desempeño docente: consideraciones desde el enfoque por competencias. Revista electrónica de Investigación Educativa. ISSN: 1607-4041 (en línea) 11(2) http://www.scielo.org.mx/scielo.php?pid=S1607-40412009000200005\&script=sci_arttex, (Noviembre 2009).

Rueda, M.; Elizalde, L. y Torquemada, A., La Evaluación de la Docencia en las Universidades Mexicanas. Revista de la Educación Superior. ISSN: 0185-2760 (en línea) 32(127), 3, julio-sep 2003, consultado 21, junio de 2012. http://publicaciones.anuies.mx/revista/127/3/5/es/la-evaluacion-de-la-docencia-en-lasuniversidades-mexicanas, (2003).

Valdés, V. H., "Encuentro Iberoamericano sobre Evaluación del Desempeño Docente". Organización de Estados Iberoamericanos para la Educación, la Ciencia y la Cultura. Desarrollo Escolar (en línea) 23 al 25 de mayo del 2000. Consultado 21 de junio 2012. http://www.oei.es/de/rifad01.htm. (2000).

Van Vaugh H., "The Humboldtian University under pressure. New forms of quality review in western European countries". London: University Open Press. (1998). 